3. Extracts of the thyroid and of the liver of hemophiliacs markedly prolong coagulation of blood.

262 South Seventeenth Street-1703 East Moyamensing Avenue.

\section{ABSTRACT OF DISCUSSION}

Dr. William Weston, Columbia, S. C.: In the treatment of these purpuric conditions, it may be well to keep in mind the marked effect that fresh fruit juices have on the coagulation of the blood. For instance, we know that in scorbutus fruit juices assist markedly in the coagulation of the blood.

\section{RECENT STUDIES IN THE ANATOMY AND PHYSIOLOGY OF. TENDONS}

\author{
THEIR APPLICATION TO THE TECHNIC OF \\ TENDON OPERATIONS *
}

\section{LEO MAYER, M.D. NEW YORK}

My interest in tendons was first awakened in the year 1912, when, while I was acting as volunteer in the clinic of Professor Lange, the problem of preventing postoperative adhesions was assigned as an experimental study to Dr. Henze of New Haven and to me.

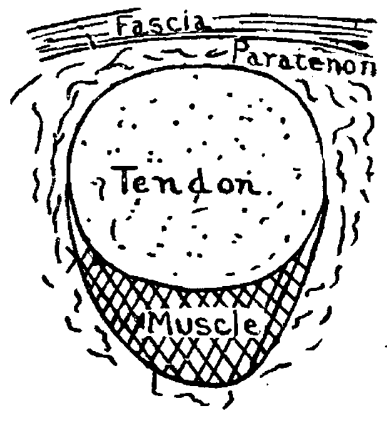

Fig. 1. Cross-section (dia. grammatic) through the tibialis anticus tendon 1 inch above the upper pole of the shearl.

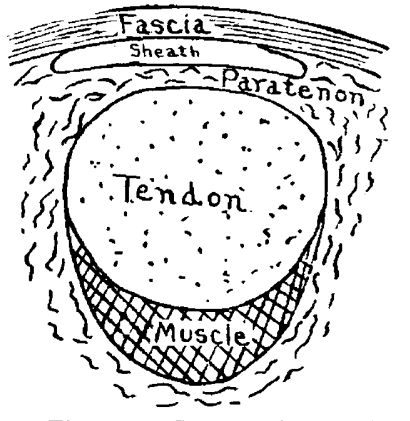

Fig. 2.-Cross-section (diagrammatic) through the tibialis anticus tendon at the level of the upper pole of the sheath.
The problem was one of great importance in Lange's eyes, since, despite his experience in 2,000 operations, the results were frequently impaired by adhesions developing subsequent to the transplantation.

It is, of course, self-evident that the function of the tendon as a means of transmitting the contraction of the muscle to the skeleton is completely inhibited by it single strong adhesion in the same way as the rope of the derrick cannot glide if clamped at a single point. In the course of our experimental investigations, which were conducted chiefly on rabbits, we utilized all manner of membrane, thin tubes of rolled silver, petrolatum, bismuth paste, fascia, peritoneum and a vein as a means of ensheathing the tendon. None of these substances, however, prevented the development of adhesions; in fact, with the exception of the Cargyle membrane, more adhesions were present after their introduction than in control experiments in which nothing was used. Finally we followed the suggestion of Biesalski and utilized the sheath of the paralyzed tendon as a physiologic pathway for the transplanted tendon; that is, one tendon was withdrawn from its sheath, cut away from the paraiyzed

* Read before the Section on Orthopedic Surgery at the Sixty-Ninth * Read before the Section on Orthopedic Surgery at the Sixty- inth
Annual Session of the American Medical Association, Chicigo, Annual Sessi Tune, 1918. muscle, and the substituting tendon drawn downward by means of a guide suture so as to occupy exactly the position of the original tendon. In all of the cases observed there was complete absence of adhesions, even when the limb was immobilized for thirty days subsequent to the operation.

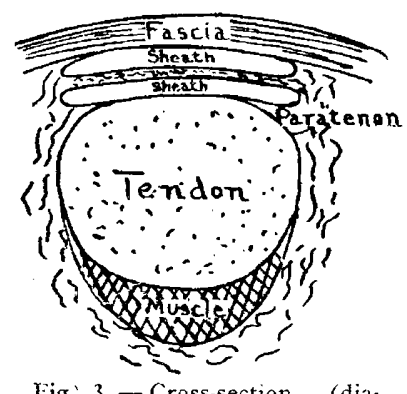

Fig. 3. - Cross-section (diagrammatic) through the tibialis anticus tendon one-half inch dis ligure 2 .

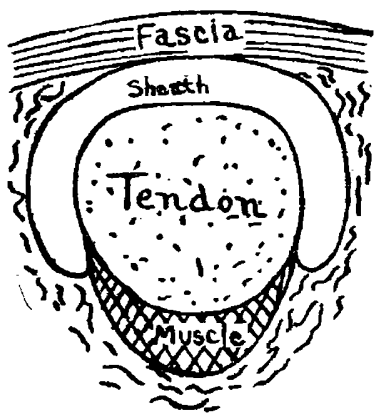

Fig. 4. - Cruss-section $11 /$ inches distal to the section shown in Figure 3.

This clear cut evidence in favor of Biesalski's method indicated to me the importance of coordinating the operative technic with the physiology of the structures involved. Just exactly as the normal relationship between tendon and sheath should be maintained, so, too, the normal fascia relationship, the normal tension and the normal fixation of the transplanted tendon should be made as nearly like the normal as possible. When, however, I tried to follow out this line of thought, I found that our knowledge of the physiology and anatomy of tendons was entirely inadequate for the purpose. No one had as yet considered the nature of the gliding mechanism of tendons. In no book, physiologic or surgical, had the subject of tendon tension ever been brought up for discussion. Despite the thousands of tendon operations, some of the simplest facts relative to their anatomy had never been investigated. It was necessary, therefore, before any comprehensive operative technic could be formulated, that these fundamental questions be investigated. The work was conducted by research on the cadaver, animal experimentation and observations on human beings.

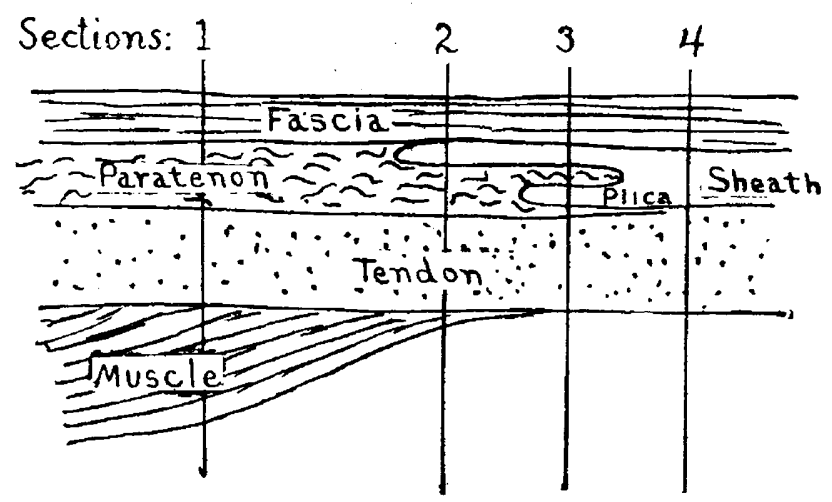

Fig. 5.-Longitudinal section (diagrammatic) of the tibialis anticus tendon, to correlate the preceding cross-sections. Note that the paratenon is prolonged downward into the sheath as a loose fold-the plica.

I can best introduce the subject of the anatomy of tendons by a series of cross-sections showing a tendon at rarious levels above and within its sheath. The first (Fig. 1) shows the tendon about 1 inch above the upper pole of the sheath: note that between the fascia and the tendon is a distinct gap not described 
in the textbooks. This space is filled with a peculiarly elastic tissue, consisting essentially of fat cells and elastic fibers. This tissue completely surrounds the tendon and the lowermost muscle fibers, and by means of its elasticity allows the tendon to glide freely to and fro beneath the rigid fascia. How great this

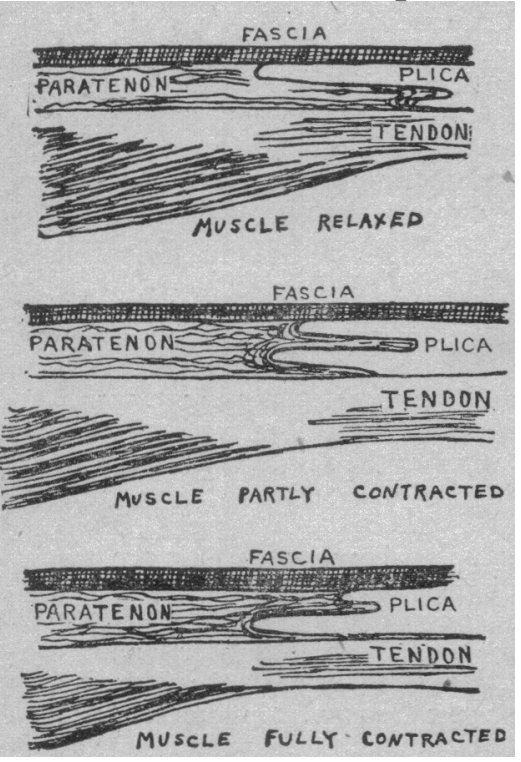

Fig. 6. - Diagrams representing the changes occurring in the form of the sheath during the contraction of the muscle and the consequent gliding of the tendon. Note that the deep pocket of the sheath between plica and tendon increases markedly in length, allowing the tendon to move upward without rupture of the sheath wall. not develop between the fascia and the tendon, but between the fascia and this gliding tissue, which I shall henceforth term the "paratenon."

In the next section (Fig. 3), which lies about half an inch distal to the preceding, the sheath is seen to be divided into two portions by a transverse band, the tissue of which is microscopically identical with the paratenon.

In the fourth cross-section (Fig. 4), about $1 \frac{1}{2}$ inches distal to the preceding, the tendon sheath is interposed between fascia and tendon and is seen almost to surround the latter. A longitudinal section (Fig. 5) enables us to correlate these four crosssections. It is evident that the paratenon is prolonged downward into the sheath as a tonguelike structure. The significance of this is appreciated only when the muscle contracts and the tendon moves upward; then it is seen that a kind of invagination occurs (Fig. 6). The deep pocket of the sheath becomes much elongated, thus allowing the tendon to glide, without destroying the continuity of the sheath wall. The essential, of course, is the elasticity of the paratenon which allows this degree of stretching.

By means of simple experiments on dogs the normal tension of tendons was accurately determined. The method consisted in the division of the tendon shortly above its insertion. The tendon ends separated for a distance of from 1 to $2 \mathrm{~cm}$. because of the tension to which they were subjected by the muscular pull; by means of a recording instrument, the proximal tendon stump was pulled downward until brought into apposition with the distal. The degree of force represented the tension to which the tendon was subjected. The figures varied markedly, according to

the varying conditions of the experiment, but one fact remained constant, irrespective of the size of the animal or the strength of the muscle: when under anesthesia, the limb was held in such a position that the origin of the muscle and its point of insertion were brought as near together as possible, then the tendon tension equaled 0 ; in other words, with the tibialis anticus divided and the foot held in the position of calcaneovarus, then under anesthesia the two tendon ends came into exact approximation without the use of any force whatever. This simple physiologic fact is readily applicable to tendon transplantation. The normal tension is restored by holding the limb in such a position as to approximate the origin of the muscle and the new site of tendon implantation.

I can best illustrate the application of the physiologic principle by outlining a typical tendon transplantation; for instance, the transfer of the extensor proprius hallucis for the paralyzed tibialis anticus. The first incision exposes the insertion of the paralyzed tendon. The tendon itself is slit longitudinally and the subjacent bone is traumatized. Against this traumatized surface the transplanted tendon is to be brought since the resulting osteogenic activity of the periosteal cells anchors the transferred tendon firmly in place.

The second incision runs along the extensor proprius hallucis. Not the entire tendon but only the upper portion is exposed at first. The idea is that the tendon should be
protected against drjing until everything is ready for its transfer. When the sheath of the extensor proprius hallucis is opened, it will be seen that it is separated from the sheath of the tibialis anticus by a fascial septum; by prolonging the incision a short distance upward one $D$ reaches a point where this septum stops short and the two tendon sheaths are separated only by a paratenon. At this point a small opening is made directly into the sheath of the tibialis anticus; a probe containing a guide suture is passed downward through the sheath and emerges just over the inser tion of the para lyzed tendon. By means of this guide suture the extensor proprius hallucis tendon,

Fig. 7.-Typical physiologic tendon transplanta. tion-transfer of the tibialis anticus for paralytic clubfoot. The probe passing through the sleath of the extensor longus digitorum emerges at the insertion of the peroneus tertius and serves to draw the tibialis tendon downward through the sheath of the paralyzed evertors. $A$, retracted fascia; $B$ to $D$, probe passing through sheath of extensor longus digitorum; $C$, tibialis anticus.

which is rapidly freed, is drawn downward through the sheath of the tibialis anticus and anchored firmly in the bed already prepared for it. In fastening it, the foot is held in the position of calcaneovarus and the tendon is pulled on with just enough force to render its course a straight one. Immobilization should not be continued more than three week.s 
because by that time firm union has occurred. The patient is then allowed to exercise the transplanted tendon, and three weeks later to walk with the transplanted tendon protected from undue tension by an appropriate splint.

The therapeutic results achieved by this method, in the 250 cases in which the operation has been performed in the last three and one half years, have been unusually gratifying.

This brief summary of the principles underlying the physiologic method indicates only in a rough way its application to the treatment of poliomyelitis and paralysis due to gunshot injuries. For further details the reader is referred to my book ${ }^{1}$ and to a series of articles published in Surgery, Gynecology and Obstetrics. ${ }^{2}$ Experience since that time, although it has altered some details, has confirmed me in the view that this method of tendon transplantation offers a valuable means of helping our patients.

149 West Seventy-Ninth Street.

\section{ABSTRACT OF DISCUSSION}

Dr. Arthur Steindler, Iowa City, Jowa: I wish to emphasize the importance of the physiologic method of tendon transplantation. Dr. Mayer said something about the clinical results. I can corroborate his statements by my own results, although they were not obtained entirely by his method. I made use of the principles mentioned in a series of about forty cases. The conclusion I can draw is that if the physiologic conditions are observed carefully, if the tendon sheath is either left unharmed, as he does, or reconstructed, as I am doing, according to an earlier suggestion of his, for which I am glad to give him credit; and, furthermore, if the vitality of the tendors is not endangered, and that is the only point, there is no failure of the tendon to work. I mean that it will act from a biologic point of view. I made a survey of my cases and found that it acted in almost every case, barring some cases of infection. With regard to the functional value, it acted functionally satisfactorily in 80 per cent., which is absolutely the converse of what I had found before using this method. One thing which makes me a little doubtful is the question of the dissociation of function. I saw a case in which a tendon transplantation had been performed several years previously and, I believe, successfully, because the tendon acted. After seven years it was, however, impossible for the patient to dissociate function between the two peronei tendons.

Dr. Eтhan H. Smitr, San Francisco: I should like to emphasize one point, and that is the outcome of tendon transplantation when there is infection. Its importance is well known to the orthopedist, but for the benefit of the general surgeon who is going to do tendon work as the result of military requirements, this point should receive particular attention. Infection is not always absent when a wound is apparently healed; we should be very certain that there is no latent infection before attempting any work on the tendons, because there is nothing that makes so complete a wreck as an infected tendon.

Dr. Charles M. Jacobs, Chicago: In tendon transplantation two important points must be considered: first, the technic, and second, the selection of muscles. In his demonstration Dr. Mayer used the extensor hallucis pollicis to take the place of the tibialis anticus. Notwithstanding the correct technic, the result would prove a failure, because a weak muscle cannot take the place of a strong one. In my opinion, many failures of tendon transplantation are due to the fact that we do not sufficiently consider this fact.

Dr. M. A. Bernstein, Chicago: I have carried on experiments for one year on tendon transplantation, following the

1. Mayer, Leo: The Orthopedic Treatment of Gun-Shot Injuries, Jhiladelphia, W. B. Saunders Company.

2. Mayer, Leo: Surg., (rynec. and Obst., 1916, 22, 182, 298, 472. technic of Dr. Mayer as closely as possible. My findings were as follows: In transplanting tendons into the sheath of other tendons there followed a traumatic inflammatory. reaction, manifesting itself in infiltrative and proliferative change in the endothelial lining of the sheath. This inflammatory reaction depended on the amount of tratuma of the tendon and the amount of adhesion, varying with the duration of immobilization of the limb. The microscopic findings are as follows: A proliferation of cells and an exudation of blood into the sheath, with fixation to the tendon to the peritendineum externum. I believed that adhesions, if permitted to organize, would produce ultimate fixation of the tendon. So we carried on experiments along different lines, transplanting the tendon with the sheath and the surrounding fascia, and made examinations varying from eight to twenty-one days after operation. We found, in the cases in which we transplanted the tendon with the sheath and surrounding tissue, that the proliferative change was not so extensive as in the other experiments. Even if there occurred a slight infection of the limb, the infiltrative change took place in the surrounding tissue, but did not extend down to the sheath. The question, therefore, comes up whether the sheath is essential in the transferring of tendons, and whether, if the tendon is transplanted without the sheath, through the sheath of another tendon, if the trauma brought about by rubbing the tendon over the wall of the sheath does not set up a traumatic inflammatory reaction with subsequent adhesions.

Dr. Leo Mayer, New York: Dr. Steindler and I disagree on only one point. He believes that the tissue which connects the tendon with the bone within the sheath, and through which the blood vessels run to the tendon (the mesotendon), should be kept intact in order to maintain the life of the tendon. Of course, it is a good thing to do this; but it is not absolutely necessary, because I have had a chance to observe that the tendon retains its vitality even when these blood vessels are divided. Of course, absolute adherence to physiologic principles in the other steps of the operation is required in order to secure success. The tendon must not be allowed to dry, and the time of exposure must not be more than half a minute. If everything is ready beforehand for the transfer of the tendon it is possible to accomplish this. The tendon gets enough blood vessels through the lower fibers of the muscles to keep it alive, since there is abundant anastomosis within the tendon.

Regarding the transplantation of the peroneus longus, I think that that is the best operation for paralytic valgus, since it converts a strong everter into an inverter of the foot. The trauma is more than offset by the function we secure.

Regarding infection, I endorse what Dr. Smith said. Never operate when there is infection. In the case of gunshot wounds, one should wait at least four months after the injury before operating.

In regard to the selection of the tendon, the point of $\mathrm{Dr}$. Jacobs is well, taken. Be very careful to select the right tendon. Do not send a boy to do a man's work. The extensor proprius hallucis should be transplanted for the tibialis anticus only when a strong tibialis posticus is present.

Dr. Bernstein's teports are exceedingly interesting, and I am glad that experimental work is being done to find out further details concerning the physiologic method. There is a certain amount of inflammatory reaction subsequent to the operation. If you draw a tendon down through the sheath of another tendon, there is bound to be some rubbing; but if you are careful it is not excessive. If you fix the tendon effectively you can begin active motion of the muscle three weeks after the operation and easily tear any adhesions that are forming. This occurred in one of my patients.

In operations at the knee there is no opportunity to use the sheath method. There are no tendon sheaths at the knee joint, but we can utilize physiologic principles. In the first place, we can maintain the normal tension of the tendon. The technic of doing this is simplified by inserting a few sutures in the quadriceps tendon which pull the patella upward. In the second place, you can render the course of the tendon as nearly normal as possible by not interfering with the paratenon, which surrounds the tendon. In these two respects the operation differs from that previously performed. 